\title{
Radiation Sensitivity and Growth Characteristics of an Arginine- and Uracil-Starved Culture of Escherichia coli strain $15 \mathbf{T}^{-} \mathbf{A}^{-} \mathbf{U}^{-}$
}

\author{
BY D. M. GINSBERG \\ Department of Biophysics, Walter Reed Army Institute of Research, \\ Washington, D.C., 20012; Bacteriology Department, University of Tennessee, \\ Knoxville, Tennessee, U.S.A. \\ AND J. JAGGER \\ Biology Division, Oak Ridge National Laboratory, Oak Ridge, Tennessee; \\ Bacteriology Department, University of Tennessee, Knoxville, Tennessee, U.S.A.
}

(Received 17 July 1964)

\begin{abstract}
SUMMARY
A logarithmic-phase culture of Escherichia coli strain $15 \mathbf{T}^{-} \mathbf{A}^{-} \mathbf{U}^{-}$, in which protein and RNA syntheses were inhibited for $90 \mathrm{~min}$. by withdrawal of arginine and uracil, was compared with a culture grown with all required nutrients present until 90-120 min. after the end of exponential increase in extinction (early stationary phase). The two cultures were indistinguishable by tests of radiation (ultraviolet, gamma) sensitivity, growth kinetics and morphology. It is concluded that the interruption of protein and RNA syntheses during active logarithmic growth forced the culture into the stationary phase.
\end{abstract}

\section{INTRODUCTION}

In the preceding paper (Ginsberg \& Jagger, 1965) we showed that the ultraviolet (u.v.) resistance of Escherichia coli strain $15 \mathrm{~T}^{-} \mathrm{A}^{-} \mathrm{U}^{-}$(15 tau) harvested in early stationary phase was identical with that of a logarithmic-phase culture in which arginine and uracil were withheld for $90 \mathrm{~min}$. (-AU culture); furthermore, the - AU and early stationary-phase cultures did not differ from each other with respect to the nature or the number of initial u.v. lesions produced by a given u.v. dose. This suggests that organisms of a - AU culture might be in a physiological state similar to that of organisms from the early stationary-phase culture. The present paper gives evidence that the $-\mathbf{A U}$ culture and the early stationary-phase culture also did not differ from each other by tests of radiobiological response, growth kinetics, or morphology. It is concluded that interruption of protein and RNA synthesis in logarithmic-phase organisms of this strain forced them into the stationary phase.

\section{METHODS}

Bacteria, media and conditions of growth, and u.v. irradiation techniques were as described in the preceding paper.

Gamma irradiation. For gamma irradiation, organisms were washed and diluted in 0.067 M-phosphate buffer $\left(6 \mathrm{~g} . \mathrm{Na}_{2} \mathrm{HPO}_{4}, 2 \cdot 3 \mathrm{~g} . \mathrm{KH}_{2} \mathrm{PO}_{4}\right.$ per l. distilled water; 
final pH 7). Gamma irradiation was performed on $5 \mathrm{ml}$. volumes of phosphatebuffer suspension of organisms in $5 \times 100 \mathrm{~mm}$. Pyrex test tubes. The gamma-ray source was a ${ }^{\circ 0} \mathrm{Co}$ 'Gammacell' Model 220 (Atomic Energy of Canada, Ltd), containing approximately 1200 curies of ${ }^{\circ} \mathrm{Co}$ (principal photon yield/disintegration: $\gamma_{1}, 1.17 \mathrm{meV} .,>99 \% ; \gamma_{2}, 1.33 \mathrm{meV} .,>99 \%$ ) distributed in standard (U.S. Atomic Energy Commission) pencils around the cylindrical irradiation chamber. During irradiation, dose-gradient effects in the irradiation chamber were minimized by constant rotation of the sample tubes around the vertical axis of the cylinder while each sample tube revolved about its own vertical axis. All irradiations were done in air at room temperature. The exposure rate was approximately 1650 roentgens/ min.

Microslide cultures for phase-contrast microscopy. Microslide cultures were prepared by the method of Adler \& Hardigree (1964), with a drop of bacterial suspension containing about $10^{8}$ bacteria/ml., which was flattened by a coverslip on to Difco nutrient agar. Growth and reproduction of individual bacteria could be observed for several hours by using the X97 oil-immersion objective of a Spencer AO phase (medium-dark contrast) microscope.

\section{RESULTS}

\section{Photobiological similarity of $-A U$ and stationary-phase cultures of Escherichia coli strain $15 \mathrm{~T}^{-} \mathrm{A}^{-} \mathrm{U}^{-}$}

In the preceding paper (Ginsberg \& Jagger, 1965) it is reported that both the u.v. survival and photo-reactivation curves were the same for - AU and early-stationary cultures of Escherichia coli strain $15 \mathrm{~T}^{-} \mathrm{A}^{-} \mathrm{U}^{-}$. Further confirmation of their photobiological similarity was obtained by the following experiment. Bacteria from each of the two cultures were inoculated into fresh growth media $(+\mathrm{T}+\mathrm{AU})$ and grown at $37^{\circ}$ for $30 \mathrm{~min}$. to mid-lag phase (half the time required for the beginning of exponential increase in viable numbers). Figure 1 shows: (1) the u.v. sensitivity of the lag-phase culture was the same whether grown from - AU or from early stationary-phase bacteria; (2) the photo-reactivable sectors were the same. The photoreactivable sectors in these experiments had the same values as for logarithmicphase, - AU and early stationary-phase cultures. The last observation shows that the photo-reactivable sector and, consequently, the spectrum of u.v. damage produced, did not change during transition from the u.v.-resistant (stationary-phase or - AU) to the u.v.-sensitive (logarithmic-phase) state. It also appears that the lag-phase survival curves had the same final slopes as the curves for logarithmicphase, - $\mathbf{A U}$, and stationary-phase cultures, suggesting that the nature of the initial lesions did not change during lag-phase transition, at least for the higher doses. The value of the ordinate intercept of the extended straight line portion of the lagphase curves is about 20, intermediate between those for logarithmic-phase cultures (5) and - AU stationary-phase cultures (273).

\section{Effects of ionizing radiation}

Billen (1963) reported the increased resistance of - AU cultures of Escherichia coli strain $15 \mathrm{~T}^{-} \mathrm{A}^{-} \mathrm{U}^{-}$to $250 \mathrm{k.v} . \mathrm{p}$. X-rays. The following experiments were done to determine whether the gamma-ray survival curves were the same for $-\mathbf{A U}$ and 
the early stationary-phase cultures. Bacterial suspensions in phosphate buffer were exposed in the ${ }^{\circ 0} \mathrm{Co}$ Gammacell. Survivors were assayed by colony formation at $37^{\circ}$ on nutrient agar; the results are shown in Fig. 2; the survival curve of a logarithmicphase culture is included for comparison. The same visually fitted curve describes the data for gamma-ray inactivation of either early stationary-phase or - AU cultures. The dose-reduction factor for early stationary-phase and - AU cultures compared to the logarithmic culture was constant (about $0 \cdot 50$ ) in the survival range studied.

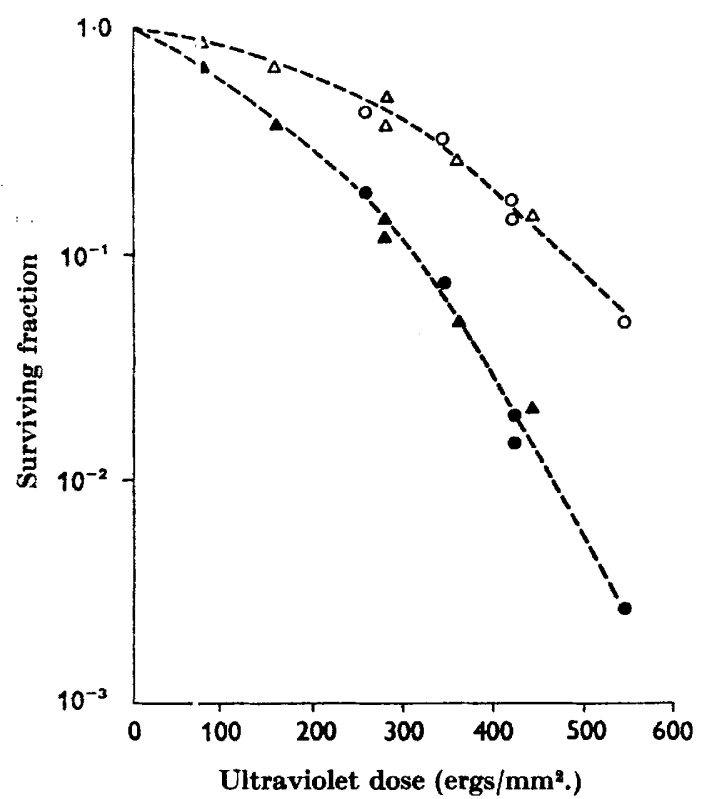

Fig. 1

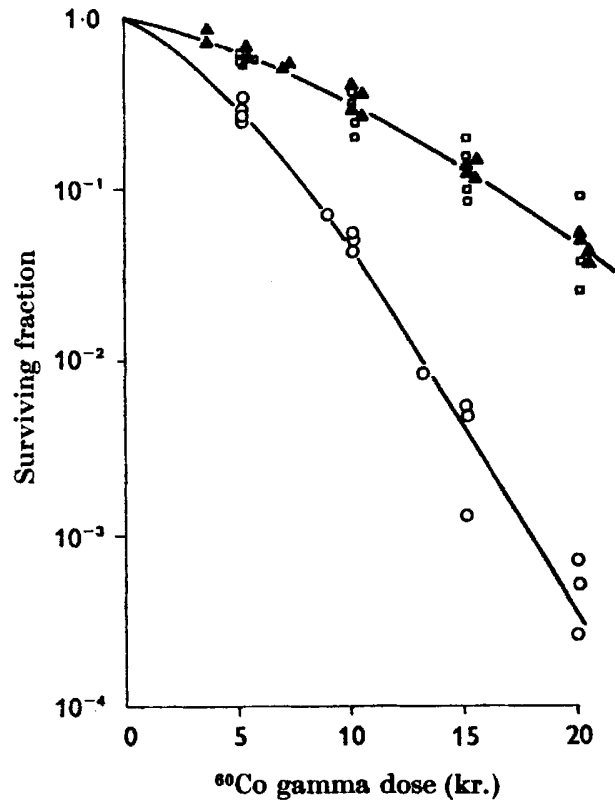

Fig. 2

Fig. 1. Survival of $E$. coli strain $15 \mathrm{~T}^{-} \mathrm{A}^{-} \mathrm{U}^{-}$after 2587 A ultraviolet-irradiation (solid symbols) and after ultraviolet + maximum photo-reactivation (open symbols) of mid-lag phase cultures grown from early stationary-phase cultures (circles) and from - AU cultures (triangles).

Fig. 2. Survival of $E$. coli strain $15 \mathrm{~T}^{-} \mathrm{A}^{-} \mathrm{U}^{-}$after ${ }^{\circ 0} \mathrm{Co}$ gamma-irradiation of logarithmicphase cultures (circles), early stationary-phase cultures (triangles), and - AU cultures (squares).

\section{Growth studies}

Growth of a logarithmic-phase culture of Escherichia coli strain $15 \mathrm{~T}^{-} \mathrm{A}^{-} \mathrm{U}^{-}$(in $+\mathbf{T}+\mathrm{AU}$ supplemented medium) was interrupted at a viable count of $10^{8}$ bacteria/ $\mathrm{ml}$. by withdrawal of arginine and uracil for $90 \mathrm{~min}$. At the end of this time, growth was permitted to resume by adding arginine and uracil. The effects of these shifts on the curves of extinction and viable count are recorded in Fig. 3. Similar results were reported by Maaløe \& Hanawalt (1961). Figure 4 shows the extinction and the viable count for $\mathbf{a}+\mathbf{T}+\mathbf{A U}$ culture entering the stationary phase and, subsequently, an early stationary-phase culture $(90 \mathrm{~min}$. after cessation of exponential extinction increase) beginning exponential growth. The similarities between Figs. 3 and 4 are striking. The slope of the extinction curve diminished abruptly when 
arginine and uracil were withdrawn from a logarithmic-phase culture, and when a normal $(+\mathbf{T}+\mathbf{A U})$ culture entered the stationary phase. Extinction increased at a high rate almost immediately when arginine and uracil were added back to the - AU culture and when early stationary-phase bacteria were inoculated into fresh growth medium. The curve of viable numbers gradually decreased in slope after arginine and uracil were withdrawn from a logarithmic culture and as the $+T+A U$ culture entered the stationary phase. Resumption of exponential increase in viability was delayed for about $60 \mathrm{~min}$. after arginine and uracil were added back to the - AU culture, and after early stationary-phase bacteria were inoculated into fresh growth medium.

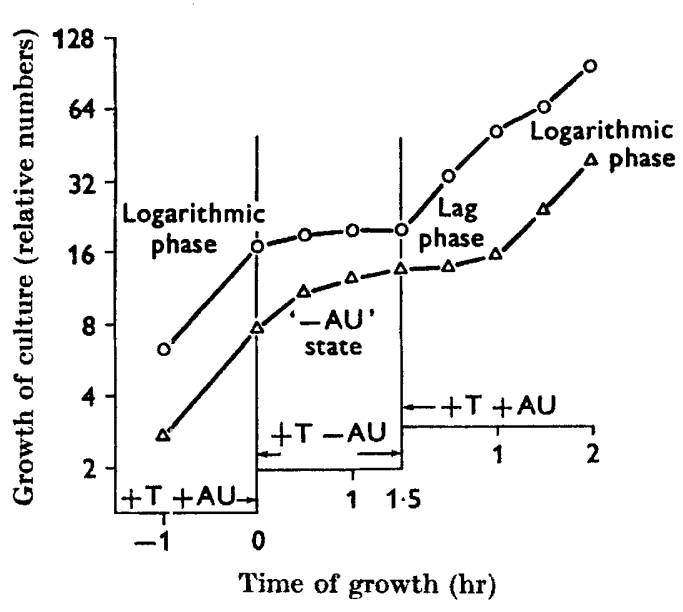

Fig. 3

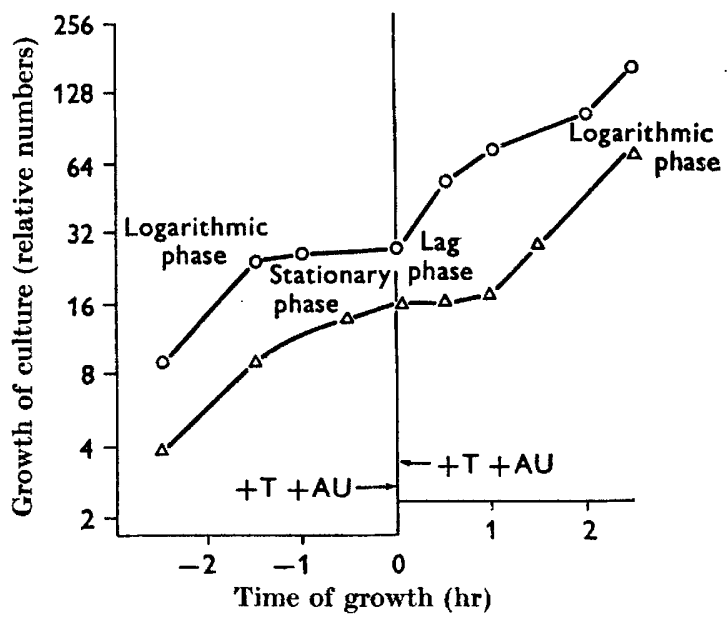

Fig. 4

Fig. 3. Growth of $E$. coli strain $15 \mathrm{~T}^{-} \mathrm{A}^{-} \mathrm{U}^{-}$, as reflected by viable numbers (triangles) and extinction (circles). A culture in the logarithmic phase was interrupted by withdrawal of arginine and uracil for $90 \mathrm{~min}$.

Fig. 4. Growth of $E$. coli strain $15 \mathrm{~T}^{-} \mathrm{A}^{-} \mathrm{U}^{-}$, as reflected by viable numbers (triangles) and extinction (circles). A culture in supplemented ( $+\mathbf{T}+\mathbf{A U})$ medium is shown during transition from logarithmic to early stationary phase, and from early stationary phase back to logarithmic phase, the latter being obtained by dilution at time zero into fresh medium.

\section{Morphology of unirradiated organisms}

Organisms from the early stationary-phase culture, and from a - AU culture, were inoculated on microslide nutrient agar mounts and observed for several divisions at room temperature by phase microscopy. No initial morphological differences were detectable between the organisms of the two cultures. The time course of increase in size during the lag phase and the time of onset of cell division were parallel in the two preparations. Initially the organisms were short, rod-shaped; the length:diameter ratio was about three. Double organisms were present in both preparations, but single organisms were predominant. In about $2 \mathrm{hr}$, organism length had doubled and a few organisms had begun to divide. At $3 \mathrm{hr}$, many organisms were about four times the original length, and more organisms were beginning to divide. By $4 \frac{1}{2} \mathrm{hr}$, microcolonies of 4-8 organisms each had formed, and almost all organisms were dividing. Central bodies were observed in most instances; and their number always appeared to be proportional to organism length. 


\section{DISCUSSION}

We have reported in the preceding paper (Ginsberg \& Jagger, 1965) that - AU and early stationary-phase cultures of Escherichia coli strain $15 \mathrm{~T}^{-} \mathrm{A}^{-} \mathrm{U}^{-}$were photobiologically alike. It is shown in the present paper that the - AU and early stationary-phase cultures returned to the u.v.-sensitive state in the same way when regrown in $+T+A U$ medium, and that both cultures showed the same degree of sensitivity to gamma radiation. The data on growth and morphology indicate that the two cultures are identical. Thus, we conclude that inhibition of protein and RNA syntheses in the logarithmic bacterial culture forced the culture into stationary phase. Although the evidence presented (growth kinetics, morphology, radiation sensitivity) is sufficient to confirm our preliminary suggestion that a - AU culture is in a physiological state similar to that of the early stationary phase, it does not provide significantly new information about this state. We still do not understand why or how the usual transition from logarithmic to stationary phase is initiated (see Freter \& Ozawa, 1963). We do not know for certain what changes, if any, occur in the quality and quantity of DNA in a bacterium entering stationary phase (Ginsberg, 1963). We have made a preliminary observation (unpublished) that the lag phase in a liquid subculture is about $20 \mathrm{~min}$. longer for a 3-4 $\mathrm{hr}$ stationary-phase culture than for the 90-120 min. culture, and it is possible that the radio-sensitivities of cultures in these two portions of the stationary phase are different. Consequently, conclusions based on the experimental observations presented here, about the nature of a stationary-phase culture, would be highly speculative. The extent and detail of the gamma-ray experiment were limited to showing that the - AU and early stationary-phase cultures are alike in their survival response to ionizing radiation. However, the appearance of a constant dose-reduction factor over the survival range tested permits us to suggest, cautiously, that a situation analogous to that for u.v. killing could exist, namely, that (1) the initial damage by a given gammaray exposure is independent of growth phase or metabolic state, and, therefore, (2) the difference in survival is the result of differences in ability to cope with the damage. This notion is consistent with the experimental findings which led Glinos \& North (1963) to conclude that a given $X$-ray dose produces the same initial damage in mammalian cells in logarithmic and in stationary-phase, even though the stationary-phase cells show a higher survival.

Based on part of a dissertation submitted by D. M. Ginsberg in partial fulfilment of the Doctor of Philosophy degree, The University of Tennessee, Knoxville, Tenessee. Research sponsored in part by the U.S. Atomic Energy Commission under contract with the Union Carbide Corporation.

\section{REFERENCES}

Adler, H. I. \& Hardigree, A. A. (1964). Analysis of a gene controlling cell division and sensitivity to radiation in Escherichia coli. J. Bact. 87, 720.

Biluen, D. (1963). Unbalanced deoxyribonucleic acid synthesis: its role in X-ray induced bacterial death. Biochim. biophys. Acta, 72, 608.

Freter, R. \& Ozawa, A. (1963). Explanation for limitation of populations of Escherichia coli in broth cultures. J. Bact. 86, 904. 
Ginsserg, D. M. (1963). The influence of metabolic state and growth phase on ultraviolet killing of Escherichia coli strain $15 \mathrm{~T}^{-} \mathbf{A}^{-} \mathbf{U}^{-}$. Ph.D. Thesis, University of Tennessee.

Ginsberg, D. M. \& JAGger, J. (1965). Evidence that initial ultraviolet lethal damage in Escherichia coli strain $15 \mathrm{~T}^{-} \mathrm{A}^{-} \mathrm{U}^{-}$is independent of growth phase. J.gen. Microbiol.40, 171.

Grinos, A. D. \& North, H. (1963). Cellular growth and tissue sensitivity: cell studies in vitro and general concepts. Trans. N.Y. Acad. Sci. 26, 145.

Maaløe, O. \& Hanawalt, P. C. (1961.) Thymine deficiency and the normal DNA replication cycle. I. J. molec. Biol. 3, 144. 